\title{
Interacción comunicativa en salud. Comprensión de conceptos mínimos en pacientes oncológicos que asisten al Centro Asistencial Barros Luco (CABL)
}

\author{
Communicative interaction in health. Compression of minimal concepts in cancer patients \\ attending the Barros Luco Healthcare Center (CABL)
}

\author{
Sabina Canales Urrutia ${ }^{1}$, Macarena Céspedes ${ }^{1}$
}

\begin{abstract}
Resumen
El presente estudio se centra en la importancia que tiene la interacción comunicativa entre médico y paciente durante el tratamiento del cáncer. El peso de la interacción recae en la comunicación efectiva desarrollada en las prácticas lingüísticas dentro de la medicina clínica. Aquí se aborda la comunicación en salud y se explica el grado de comprensión que el paciente oncológico tiene en torno a cuatro conceptos elementales: cáncer, metástasis, tipos de tumores y tipos de terapia, los que se expresaron para ellos en tres tipos de definiciones: técnica, acomodada y coloquial. Para esto se aplicó una encuesta sociolingüística mixta a 50 pacientes en el Hospital Barros Luco, en donde se observó que más del 50\% de los pacientes comprende toda la información cuando esta se explica de manera técnica y, más del $70 \%$ incluso $80 \%$ comprende toda la información cuando se explica de una manera acomodada a la valoración del contexto, situación e interlocutores. Los resultados también indican que los médicos, según los pacientes entrevistados, explican con un lenguaje de código cerrado y, en ocasiones, simplemente no entregan la información. Según los resultados se puede concluir que la información previa junto con la experiencia comunicativa, entendida como una práctica social, ayudan a construir y representar una nueva realidad sociolingüística que coopera para que los pacientes oncológicos logren entender la información verbal, así como también el nuevo contexto social en el que se encuentran.
\end{abstract}

Palabras clave: interacción comunicativa; comprensión; paciente oncológico; comunicación en salud; sociolingüística.

\begin{abstract}
The present study focuses on the importance of the communicative interaction between doctor and patient during cancer treatment. The weight of the interaction falls on the effective communication developed in linguistic practices within clinical medicine. Here, health communication is addressed, and the degree of understanding that the cancer patient has around four elementary concepts been explained: cancer, metastasis, types of tumors, and types of therapy, which were expressed for them in three types of definitions: technical, well-off and colloquial. For this, a mixed sociolinguistic survey was applied to 50 patients at the Barros Luco Hospital, where it was observed that more than $50 \%$ of patients understand all the information when it is explained technically, and more than $70 \%$ even $80 \%$ understand all the information when it is explained in a way adapted to the assessment of the context, situation, and interlocutors. The results also indicate that the doctors, according to the patients interviewed, explain with a closed code language and sometimes, simply do not provide the information. According to the results, it can be concluded that the previous information together with the communicative experience, understood as a social practice, help to build and represent a new sociolinguistic reality that cooperates so that cancer patients can understand verbal information, as well as the new context social where they are.
\end{abstract}

Keywords: Communicative interaction; understanding; cancer patient; health communication; sociolinguistics.

Fecha de envío:2020-06-08 - Fecha de aceptación: 2020-12-18

(1) Departamento de Lengua y Literatura, Facultad de Filosofía y Humanidades, Universidad Alberto Hurtado. Autor de correspondencia: sabina.canales.urrutia@gmail.com 


\section{Introducción}

Dentro de la oncología, la interacción comunicativa entre médico y paciente es compleja: por un lado, implica el dominio de un léxico altamente especializado, difícil tanto de comprender como de distinguir en la forma de la palabra -un término de otro- $y$, por otro, la implicancia de lo que transmite su significado por la gravedad de la enfermedad, así como la carga pragmática que esta tiene por ser la segunda causa de muerte en Chile según el Plan Nacional del Cáncer (2018-2028). Esta investigación reflexiona en torno a la medicina clínica, principalmente, sobre su actuar en la interacción comunicativa a fin de obtener resultados que beneficien al paciente oncológico en el tratamiento del cáncer y todo el proceso que esto implica.

La presente investigación se basa en el método sociolingüístico desde el cual se analiza el cruce entre lenguaje y sociedad, para explicar la complejidad de la interacción comunicativa humana. Nuestra tarea, aquí, es aportar con un análisis de lenguaje y acción social aplicado a la medicina clínica, identificando las necesidades comunicativas e intervenir en diferentes contextos en donde interactúan los principales actores, a saber, médicos y pacientes. Desde esta mirada, el objetivo de la investigación se centra en explicar el grado de comprensión que estos últimos tienen frente a conceptos oncológicos elementales y cómo reciben la información durante la interacción comunicativa en la consulta médica.

Según lo anterior, el paciente necesita comprender las condiciones en las que se genera la interacción comunicativa, como la situación y el contexto para acomodarse al tipo de lenguaje que usa el médico tratante, que para él significa un nuevo lenguaje. Este hecho se explica bajo el modelo de acomodación lingüística que intenta explicar los cambios en los estilos de habla como resultado del contexto y situación comunicativa (Moreno-Fernández, 2012) desde el cual los hablantes se enfrentan a un nuevo lenguaje en una nueva comunidad. En este sentido, tanto el médico como el paciente deben ajustar su registro lingüístico para lograr una comunicación y comprensión efectiva en la consulta clínica.

El concepto de comunicación, desde una perspectiva clínica (Carrato, 2007), debe ser entendido como todo aquello que rodea la interacción comunicativa, comprender la percepción de la enfermedad que es diferente para cada paciente, el nuevo proceso que debe enfrentar, la alimentación, los tratamientos que puede tomar y los que no. De este modo, la persona enferma de cáncer construye una representación mental del entorno comunicativo (Parodi, 2008), en donde se involucran los interlocutores: médico-paciente, junto con el contexto: servicio de oncología del Hospital Barros Luco, en este caso.
En Chile, existe un vacío de conocimiento sobre la interacción comunicativa en oncología. Esto se puede salvar interviniendo los dos sistemas de salud que existen en Chile: público y privado, para conocer la interacción comunicativa en salud que puede variar en cada grupo social. En este sentido, el presente escrito pretende dar voz a los pacientes que asisten al servicio público para que entreguen su opinión de lo que comprendieron durante su proceso en la enfermedad.

Para llevar a cabo la investigación se intervino al Complejo asistencial Barros Luco (en adelante CABL), únicamente, se muestreó a los pacientes oncológicos. Este hospital está ubicado en la comuna de San Miguel y recibe a las comunas del sector sur de Santiago. Se seleccionó este contexto de estudio: sistema de salud público, porque recibe al $80 \%$ de la población chilena y estas características de densidad demográfica de pacientes lo convierte en un contexto comunicativo de alta complejidad interaccional. Con los resultados que aquí se comunican se intenta conocer la opinión del paciente desde la necesidad de estar informado con un lenguaje cercano y comprensible, tal como se enmarca en la ley 20.584 que regula los derechos y deberes que tienen las personas en relación con acciones vinculadas a su atención en salud en donde se especifica el derecho a la información ( $5^{\circ}$ párrafo) (Ministerio de Salud; Subsecretaria de Salud Pública, 2012).

\section{Materiales y métodos}

Dentro de la sociolingüística existe un "enfoque analítico basado en la observación de la producción conectada con el contexto social de los hablantes" (Caravedo, 2014) y se convierte en el punto de partida para comenzar a investigar los diferentes contextos comunicativos que influyen en la decisión de cada hablante al momento de comunicarse. Para estos fines, la observación preliminar de corte etnográfico se realizó en la sala de espera del CABL entre los días 8 y 12 de octubre del 2018, en donde se observó a los pacientes que intercambiaban información sobre sus médicos tratantes, el trato de los profesionales de la salud, los efectos secundarios de los tratamientos y qué hacían para solucionarlos. Esta observación sirvió para realizar un análisis preliminar de las necesidades lingüísticas de los pacientes y así realizar una encuesta que ofreciera resultados sobre el tipo de información que se les entrega a los pacientes y la estrategia comunicativa que emplean los profesionales para comunicarse.

\section{Creación del instrumento}

Se creó una encuesta sociolingüística mixta cuyos datos fueron procesados en el programa Statiscal Package for the Social Sciences (SPSS). Esta encuesta consta de 2 partes. La primera consiste en 4 
preguntas de percepción en donde se mantuvo una conversación con el paciente quien reflexionó acerca de la interacción comunicativa con su médico tratante, su proceso de cáncer y su opinión en torno a la entrega de información otorgada por los profesionales de la salud. Estas conversaciones fueron registradas por medio de una grabadora de voz $y$, posteriormente, transcritas y codificadas para futuros análisis discursivos los que no serán informados en el presente escrito. La segunda parte cuenta con 2 secciones a modo de cuestionario en donde cada participante marcaba sus respuestas. En la primera sección se recogen datos personales del paciente, como: género, edad, nivel educacional, tipo de cáncer, etapa clínica del cáncer y tipo de tratamiento. La segunda sección recoge el grado de comprensión adquirido por el paciente, mediante una serie de preguntas cerradas en las que se incluyen tres formas distintas de explicar cuatro conceptos elementales. La primera explicación es la más técnica que se obtuvo del Instituto Nacional del Cáncer de
Chile (www.incancer.cl) y de Estados Unidos (www.nih.gov). La segunda forma es acomodada aplicando el modelo de la acomodación comunicativa, donde el hablante, médico tratante, adaptaría sus estrategias comunicativas y decide acomodar su registro de uso dependiendo del contexto socio-situacional. Finalmente, la explicación coloquial indica una definición informal que podría escucharse de personas que no están familiarizadas con el ámbito de la salud. Las dos últimas explicaciones fueron adaptaciones tomados de la bajada etnográfica en la sala de espera, vale decir, la observación, el registro del comportamiento, las opiniones y el uso del lenguaje expresado por una agrupación social específica, a saber, pacientes oncológicos que asisten al CABL.

El instrumento muestra cuatro alternativas para medir el grado de comprensión de cada explicación entregada, tal como se muestra en la figura 1.

c) Los cánceres pueden dispersarse por el cuerpo de dos formas: invasión o metástasis. Esta última, significa la capacidad o habilidad que poseen las células cancerígenas para viajar por los vasos linfáticos o sanguíneos y crecer en otro tejido del cuerpo que antes estaba sano.

Entiende toda la información, porque es clara y precisa.

Entiende la mayoría de los conceptos, pero no logra comprender con claridad la información

Entiende vagamente la información, porque tiene alguna idea sobre los contenidos señalados.

No entiende nada, los conceptos son muy complicados y el contenido no es claro.

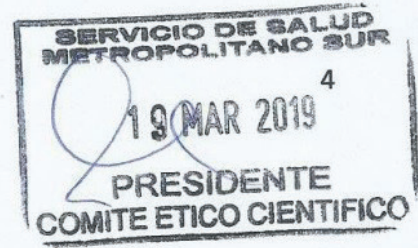

Figura 1: Ejemplo de cuatro alternativas para medir el grado de comprensión. Explicación acomodada del concepto "Metástasis".

Tras cada concepto se preguntó: “¿cuál definición cree usted que usó su médico para explicar el concepto?", esto para conocer el tipo de interlocutor con el que se enfrentó el paciente y si este recuerda la información entregada. Los conceptos que se proponen son: cáncer, metástasis, tipos de tumores y tipos de terapia. Cada concepto sigue un patrón de definición, todas las alternativas "a" corresponden a una explicación técnica. Para los conceptos 'cáncer' y'tipos de tumores' la explicación acomodada se encuentra en la alternativa "b", mientras que la explicación coloquial está en la "c". Para los conceptos 'metástasis' y 'quimioterapia y radioterapia' la explicación acomodada es la alternativa "c"y la explicación coloquial es la"b". El orden de la categoría a la que corresponden las alternativas no son conocidas por las personas encuestadas para evitar un patrón de respuestas que suele ocurrir en respuestas cerradas. La encuesta fue creada según el proceso de comprensión que deberían seguir los pacientes, a partir de la experiencia compartida por ellos, esto significa que cada concepto expuesto necesita de un conocimiento previo para crear relaciones entre las palabras, puesto que hay una pertenencia mutua entre un concepto y otro, producto de las experiencias asociativas que se vayan estableciendo (Croft \& Cruse, 2008). Así, el orden de las palabras constituye una comprensión previa para generar relación semántica, de este modo, la persona debe entender primero qué significa 'cáncer' para comprender qué es una 'metástasis'. 


\section{Aprobación del CABL y muestreo}

Para ingresar al CABL se obtuvieron todas las aprobaciones correspondientes. La primera aprobación fue desde la unidad de estudios asistencial, luego del comité de ética científico quienes entregaron la aprobación con el memorándum $N^{\circ} 122$, después el comité jurídico, en donde se firmó un convenio entre la Universidad Alberto Hurtado y el $\mathrm{CABL}$, y finalmente entregaron la resolución exenta $\mathrm{N}^{\circ} 2326$, en donde se manifiesta la última aprobación para comenzar la investigación en terreno.

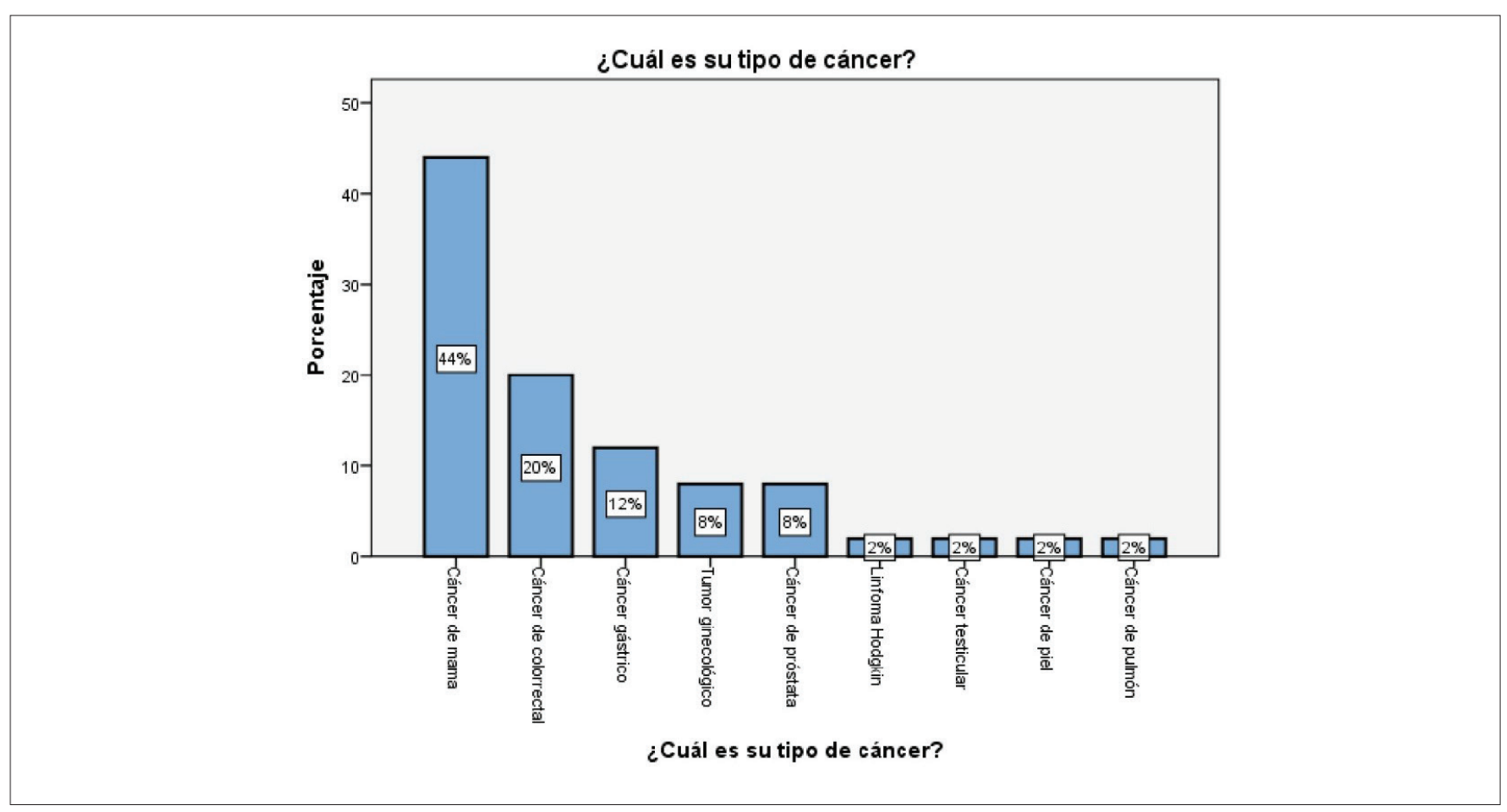

Figura 2: Tipo de cáncer.

Se realizó un muestreo al azar no probabilístico, para alcanzar una representatividad de los pacientes oncológicos con una significancia cualitativa. El criterio mínimo clínico fue contar con pacientes que llegaron al hospital a través de una interconsulta entregada por sus respectivos centros de salud primario. Cada paciente que llega al servicio de oncología del CABL sabe que está enfermo de cáncer. Como este estudio es exploratorio no distinguimos en tipos de pacientes oncológicos por lo que nuestros participantes conforman una muestra de diversos tipos de cáncer como lo informa la figura 2. Tampoco se distinguió entre estadios ni si el informante asistía a su primera consulta oncológica o no.

El muestreo se realizó en dos procesos, el primero fue un piloto a 10 personas, 5 hombres y 5 mujeres, en la sala de espera, y el segundo fue a 40 personas en un box proporcionado por la enfermera jefa del servicio de oncología. El total de la muestra fue de 50 personas, 34 mujeres y 16 hombres, todas mayores de 18 años.
Para la implementación del instrumento una de las investigadoras responsables asistía al CABL en donde se entregaba un consentimiento informado a cada sujeto para que lo leyera (en caso de analfabetismo la investigadora realizaba la lectura en voz alta), una vez tomada la decisión el paciente nos decía si participaría o no. Quienes decidían participar firmaban dos copias de este consentimiento una quedaba para archivo de nuestra investigación y la otra para el paciente. La implementación de la prueba piloto se llevó a cabo los días 22, 26, 28 y 29 de noviembre del 2018. Mientras que la prueba oficial se implementó los días 30, 31 de mayo, 5, 6, 11, $12,14,18,21,25,26,27$ y 28 de junio del 2019

\section{Resultados}

Los primeros datos tienen relación con la información personal y su enfermedad, estos aportan a la investigación el conocimiento básico del paciente sobre su entorno y la comprensión del objetivo lingüístico. La variable de la edad se muestra en la tabla 1: 
Tabla 1: Rangos de edad. Número en paréntesis corresponde a la cantidad de personas.

\begin{tabular}{lc|c}
\hline & RANGOS DE EDAD & PERSONAS \\
\hline 1. & $21-30$ & $4 \%(2)$ \\
\hline 2. & $31-40$ & $10 \%(5)$ \\
\hline 3. & $41-50$ & $12 \%(6)$ \\
\hline 4. & $51-60$ & $32 \%(16)$ \\
\hline 5. & $61-70$ & $36 \%(18)$ \\
\hline 6. & $71-80$ & $4 \%(2)$ \\
\hline 7. & $81-90$ & $2 \%(1)$ \\
\hline
\end{tabular}

Los datos que siguen hacen referencia a la información médica de los pacientes. En la figura 2 se observan 8 tipos de cánceres, siendo el de mama el que contiene el mayor porcentaje que corresponde a 22 mujeres, estos datos, confirman la información entregada por el Plan Nacional del Cáncer del MINSAL (2018-2020), donde se explica que el cáncer de mama es la principal causa de muerte en mujeres alrededor del mundo. Cabe mencionar que el cáncer de útero, de ovario y cervicouterino están agrupados en la figura 2 como 'tumor ginecológico'.

Con respecto al estadio o etapa clínica, el $28 \%$ de los pacientes contestó estar en etapa 4, tal como se observa en la figura 3:

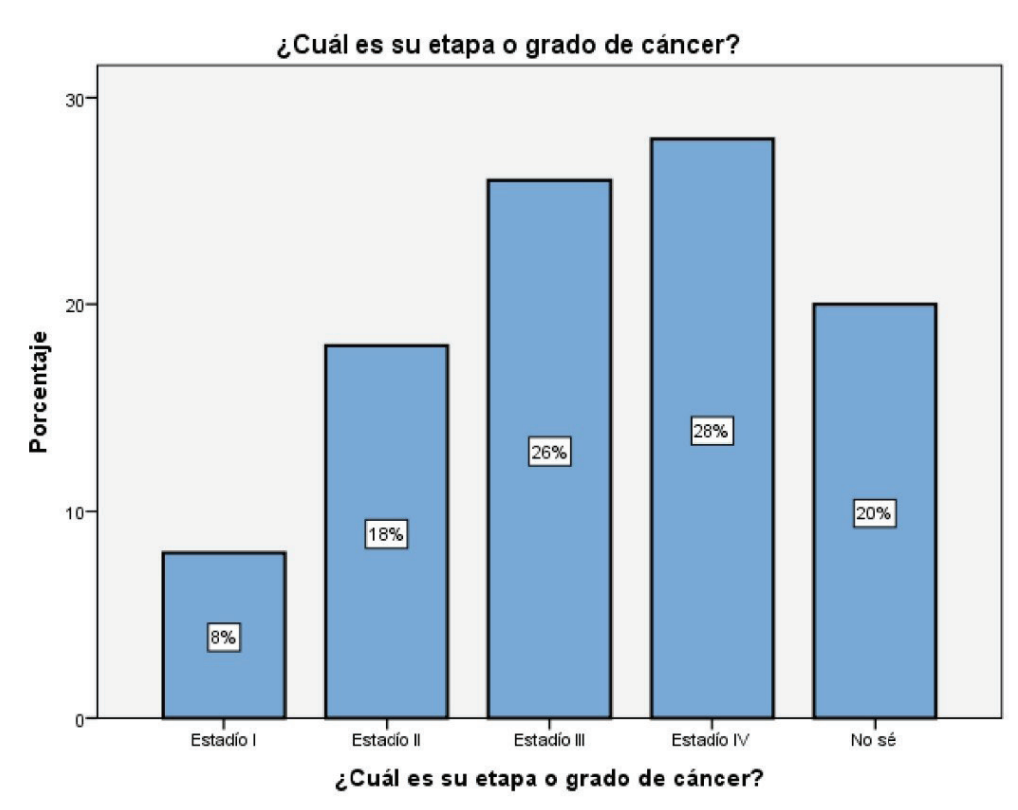

Figura 3: Etapa clínica de cáncer.

Se observa que el $20 \%$ de la muestra no conoce la etapa clínica de su cáncer, lo que deja a la persona con una ignorancia vital que resulta perjudicial para integrar más información sobre su estado; por desconocimiento o falta de comprensión acerca del término "etapa clínica". La figura 3 es parte de lo que entendió el paciente sobre su etapa clínica, ya que la investigación no tuvo acceso a fichas clínicas para corroborar la información proporcionada.

\section{Grado de comprensión sobre conceptos}

La comprensión comunicacional está contenida en dos tipos de significados: el conceptual o referencial y el pragmático-discursivo. En un contexto clínico especializado como el oncológico, la persona necesitará comprender qué es el 'cáncer', junto con asimilar qué significa estar enfermo de cáncer; lo que significaría cambiar su estilo y calidad de vida, asistir a controles sistemáticos, recibir tratamientos largos e invasivos o breves y menos invasivos según la etapa en la que se encuentre, por mencionar algunos hechos que permiten una comprensión integral de la enfermedad. De este modo, fijan en su memoria una nueva experiencia comunicativa para representar mentalmente un contexto clínico oncológico. En este nuevo contexto, el paciente comienza a relacionar lo referencial con lo pragmático-discursivo, percibiendo mediante esa relación la interpretación de la interacción comunicativa entre médico-paciente.

Según los resultados, los pacientes tienen un grado alto de compresión de los conceptos mínimos. En la tabla 2 se aprecian los porcentajes: 
Tabla 2: Opinión del paciente sobre el grado de comprensión de los 4 conceptos, entre paréntesis está el tipo de explicación que escucharon los pacientes. Número en paréntesis corresponde a la cantidad de personas.

\begin{tabular}{|c|c|c|c|}
\hline Grado de comprensión & Cáncer (técnica) & Cáncer (acomodada) & Cáncer (coloquial) \\
\hline Entiende toda la información & $52 \%(26)$ & $70 \%(35)$ & $76 \%(38)$ \\
\hline Entiende la mayoría & $16 \%(8)$ & $18 \%(9)$ & $14 \%(7)$ \\
\hline Entiende vagamente & $20 \%(10)$ & $6 \%(3)$ & $6 \%(3)$ \\
\hline No entiende nada & $12 \%(6)$ & $6 \%(3)$ & $4 \%(2)$ \\
\hline Grado de comprensión & Metástasis (técnica) & Metástasis (coloquial) & Metástasis (acomodada) \\
\hline Entiende toda la información & $64 \%(32)$ & $74 \%(37)$ & $80 \%(40)$ \\
\hline Entiende la mayoría & $8 \%(4)$ & $8 \%(4)$ & $8 \%(4)$ \\
\hline Entiende vagamente & $16 \%(8)$ & $12 \%(6)$ & $6 \%(3)$ \\
\hline No entiende nada & $12 \%(6)$ & $6 \%(3)$ & $6 \%(3)$ \\
\hline Grado de comprensión & Tipos de Tumores (técnica) & Tipos de Tumores (acomodada) & Tipos de Tumores (coloquial) \\
\hline Entiende toda la información & $72 \%(36)$ & $74 \%(37)$ & $82 \%(41)$ \\
\hline Entiende la mayoría & $16 \%(8)$ & $12 \%(6)$ & $8 \%(4)$ \\
\hline Entiende vagamente & $8 \%(4)$ & $10 \%(5)$ & $4 \%(2)$ \\
\hline No entiende nada & $4 \%(2)$ & $4 \%(2)$ & $6 \%(3)$ \\
\hline Grado de comprensión & $\begin{array}{l}\text { Quimioterapia y Radioterapia } \\
\text { (técnica) }\end{array}$ & $\begin{array}{c}\text { Quimioterapia y Radioterapia } \\
\text { (coloquial) }\end{array}$ & $\begin{array}{c}\text { Quimioterapia y Radioterapia } \\
\text { (acomodada) }\end{array}$ \\
\hline Entiende toda la información & $68 \%(34)$ & $82 \%(41)$ & $80 \%(40)$ \\
\hline Entiende la mayoría & $22 \%(11)$ & $8 \%(4)$ & $10 \%(5)$ \\
\hline Entiende vagamente & $2 \%(1)$ & $4 \%(2)$ & $4 \%(2)$ \\
\hline No entiende nada & $8 \%(4)$ & $6 \%(3)$ & $6 \%(3)$ \\
\hline
\end{tabular}

Es interesante leer que, dentro de la muestra, y de forma estimada, más del 50\% de los pacientes seleccionó la opción: "entiende toda la información porque es clara y precisa" cuando la explicación fue técnica. Mientras que un máximo de un $12 \%$ equivalente a 6 personas seleccionó la opción: "no entiende nada, los conceptos son muy complicados y el contenido no es claro". Las cifras anteriores son positivas, ya que el grado de comprensión de los conceptos es alto a pesar de recibir una explicación técnica, esto significa que el bagaje experiencial de los pacientes sobre la enfermedad colabora en la comprensión de los conceptos y de su proceso.

Después de cada concepto se preguntó “¿cuál definición cree usted que usó su médico para explicar el concepto?". Los resultados se muestran en la tabla 3 que está ordenada según el patrón de alternativas presentada en la encuesta:

Tabla 3: Opinión del paciente sobre la explicación que usó el médico tratante para definir los 4 conceptos. Número en paréntesis corresponde a la cantidad de personas.

\begin{tabular}{|c|c|c|}
\hline Definición & Cáncer & Tipos de tumores \\
\hline Técnica & $16 \%(8)$ & $18 \%(9)$ \\
\hline Acomodada & $16 \%(8)$ & $16 \%(8)$ \\
\hline Coloquial & $10 \%(5)$ & $\mathbf{2 0 \%}(10)$ \\
\hline Ninguna & $10 \%(5)$ & $4 \%(2)$ \\
\hline No hubo información & $46 \%(23)$ & $34 \%(17)$ \\
\hline No recuerda & $\mathbf{2 \%}(1)$ & $8 \%(4)$ \\
\hline Definición & Metástasis & Quimioterapia y Radioterapia \\
\hline Técnica & $\mathbf{2 4 \%}(12)$ & $\mathbf{2 2} \%(11)$ \\
\hline Coloquial & $10 \%(5)$ & $\mathbf{3 8} \%(19)$ \\
\hline Acomodada & $12 \%(6)$ & $12 \%(6)$ \\
\hline Ninguna & $10 \%(5)$ & $10 \%(5)$ \\
\hline No hubo información & $40 \%(20)$ & $16 \%(8)$ \\
\hline No recuerda & $4 \%(2)$ & $2 \%(1)$ \\
\hline
\end{tabular}


En la tabla se aprecian tres alternativas que no aparecen en la encuesta y corresponden a las tres últimas que fueron entregadas por los pacientes al no poder responder con las alternativas originales. Con respecto a'ninguna'se referían a que recibieron información, pero los profesionales de la salud habrían usado otra estrategia comunicativa, distinta a la presentada en la encuesta. Los pacientes mencionaron que, en ocasiones, el médico tratante les explicaba los síntomas de la enfermedad y descartaba la oportunidad de explicar la enfermedad en sí.

Se puede observar también altos porcentajes de desinformación, el mayor índice corresponde a la propia enfermedad con un $46 \%$, esto demuestra que la comunicación efectiva no se logró y que los pacientes averiguaron la información por otros medios, en lugar de recibir este conocimiento por su médico tratante. Mientras que para los conceptos "quimioterapia y radioterapia" solo un $16 \%$ no fue informado, esto se presenta a partir del protocolo que deben firmar los pacientes antes de comenzar los tratamientos.

\section{Discusión}

La investigación se centró en la opinión del paciente sobre su comprensión durante las consultas con su médico tratante, por ende, una de las limitaciones que tiene este estudio se relaciona con la falta de participación de los profesionales de salud. Esto se debe al poco personal especialista que se encuentra en el $C A B L$, quienes deben atender a una gran cantidad de público que asiste al servicio de oncología. De este modo, las investigadoras consideraron solo la voz del paciente en este estudio para conocer su experiencia sociocomunicativa en una situación y contexto determinado.

La experiencia directa que tengan los pacientes dentro de un contexto comunicativo clínico influye en la comprensión de los conceptos y de lo que rodea a la enfermedad. Por eso, se puede observar un mayor porcentaje de comprensión en los tipos de terapia, debido a que una colectividad comparte una experiencia y un conocimiento especializado alcanzado tras participar de una misma actividad que define a esa colectividad (Croft \& Cruse, 2008). La quimioterapia es un tratamiento largo y en las sesiones las personas permanecen en una misma sala donde comparten sus experiencias y son integrantes de una colectividad que se identifica de forma social y lingüística. Esta experiencia compartida y el cómo interprete dicha experiencia dependerá del cómo y cuánto comprenda el paciente su enfermedad.

A propósito de la figura 3 en donde se muestra el estadio de los pacientes, se puede mencionar que el desconocimiento de la enfermedad o de su etapa podría influir en un retraso en la atención del paciente. Sin embargo, quienes lo conocen, a veces, no pueden ser atendidos a la brevedad debido a ciertas limitaciones del sistema de atención púbica, generando con esto un progreso en su estadio lo que agrava y complejiza su tratamiento por parte del cuerpo médico. Por ejemplo, en el corpus de entrevistas, una informante con cáncer de piel contó que su enfermedad fue diagnosticada en estadio 2 y que esperó un año para ser atendida porque no la contactaban debido a un problema administrativo. Una vez que pudo acudir a la consulta su cáncer había aumentado a estadio 4 con metástasis pulmonar.

Se precisa que dentro de la consulta médica exista una conversación junto con una explicación fluida, esto aumentará la confianza y fortalecerá la relación entre médico y paciente. Este último se sentirá en libertad para preguntar porque el canal de comunicación va a estar abierto, lo que motiva a la persona enferma de cáncer a indagar sobre su proceso y recibir de manera positiva la información. Lograr la comprensión de los conceptos, en algunas ocasiones, puede necesitar ajustes para incorporar en la mente de los pacientes un nuevo conocimiento a partir de una nueva situación comunicativa. Como se sabe, a veces se ve que "la medicina administrativa y el economicismo de la práctica médica, son factores que entorpecen la relación médico-paciente, siendo en gran medida culpables de la pérdida de confianza que afecta a la medicina actual (Kottow, 2009)". De acuerdo con esto, el factor emocional dentro de todo el proceso de cáncer ayudará a crear un clima de confianza durante la interacción comunicativa.

Los pacientes al escuchar las diferentes explicaciones necesitan asimilar esa información y conectarla con sus redes cognitivas para lograr la comprensión. De este modo, una de las variables que influye en la comprensión y en el estado de salud de las personas son las determinantes sociales, aquello que rodea al paciente; como su trabajo, amigos, familia, estilo de vida, situación socioeconómica, nivel educacional, entre otros. Es por esto que las personas crean una red cognitiva que funciona como puente para recibir una nueva información y convertirla en un conocimiento que se fija en la memoria y se posiciona como parte de esa red que enmarca una determinada realidad.

Según los resultados obtenidos desde la percepción de los pacientes, la explicación técnica, aunque es una forma efectiva de comunicar, no es la preferida por ellos cuando su lenguaje con un código cerrado y no está adaptado al interlocutor, por lo mismo, los actores de la medicina oncológica necesitan conocer estrategias comunicativas que les permitan entregar una información de calidad, pero que a la vez sea comprensible para su interlocutor, quien es el que necesita conocer su situación y comprender lo que le está pasando. Siguiendo la teoría de la valoración (Kaplan, 2004), estas estrategias dependen de la capacidad de acomodarse a los diferentes contextos y situaciones comunicativas. Como autoras proponemos la siguiente tabla para valorar cada situación comunicativa con el fin de que los profesionales de la salud acomoden lingüísticamente su registro y estilo de habla dependiendo del proceso del paciente. 
Tabla 4: Propuesta para valorar las necesidades de los pacientes dentro de una interacción comunicativa. Esta puede cambiar a lo largo del proceso de la enfermedad. Por ello, se propone valorar el contexto y la situación comunicativa para lograr una comunicación efectiva entre interlocutores.

\begin{tabular}{|c|c|c|c|}
\hline Contexto comunicativo & Situación comunicativa & Tema & Relación entre médico y paciente \\
\hline \multirow[t]{5}{*}{ Unidad Oncológica } & $\begin{array}{l}\text { Consulta de resultado de biopsia } \\
\text { positiva }\end{array}$ & $\begin{array}{l}\text {-Qué significa una biopsia positiva } \\
\text {-Qué es el cáncer, } \\
\text {-de qué depende la gravedad de la } \\
\text { enfermedad } \\
\text { (valorar los conceptos asociados a la } \\
\text { evidencia del resultado de la biopsia) }\end{array}$ & \multirow{5}{*}{$\begin{array}{l}\text { + conocido } \\
\text { - conocido } \\
\text { + experiencia clínica de este tipo (no es } \\
\text { primera vez que se realiza una biopsia) } \\
\text { - experiencia clínica de este tipo } \\
\text { + experiencia oncológica (directa o in- } \\
\text { directa, como por amigo/s o familiar/es) } \\
\text { - experiencia oncológica } \\
\text { + nivel de instrucción educacional } \\
\text { - nivel de instrucción educacional }\end{array}$} \\
\hline & Primer control post quimioterapia & \multirow{4}{*}{$\begin{array}{l}\text { Aquí se pueden establecer los temas } \\
\text { a tratar en cada caso, valorando la } \\
\text { situación comunicativa y la relación } \\
\text { con los interlocutores (pacientes tipo). } \\
\text { Comprendiendo al paciente desde un } \\
\text { paradigma sicológico y social }\end{array}$} & \\
\hline & Quinto control post quimioterapia & & \\
\hline & Primer control post operatorio & & \\
\hline & Etc. & & \\
\hline
\end{tabular}

En la tabla se indica un contexto y situación comunicativa. El primero tiene relación con factores externos implicados en la comunicación, como es el lugar, una dimensión espacial y temporal. La segunda, tiene que ver con el tema que se va a tratar en la interacción comunicativa, es por ello que se ofrecen ejemplos de temas que podrían ser tratados en una consulta clínica. A partir de cada tema el médico podría acomodar lingüísticamente la información que va a entregar para hacer efectiva la comunicación. Aquí es cuando la teoría de acomodación lingüística toma fuerza, dado que a partir de la valoración que haga el profesional de la salud sobre el contexto y la situación comunicativa podrá reflexionar sobre el qué dirá, cómo lo dirá, el tono que usará, el registro y estilo que empleará durante la interacción comunicativa. En el apartado"relación entre médico y paciente" se propone valorar las determinantes sociales de cada paciente y la experiencia en el contexto comunicativo, así también, si entre interlocutores es la primera vez que tienen contacto, eso también incidiría en la relación del médico y paciente. De esta forma, una escucha activa por parte de los profesionales de la salud ayudará a conocer las necesidades comunicativas y el proceso por el cual está pasando el paciente.

La comunicación en salud es un tema que se ha estudiado desde diferentes enfoques, dada la amplitud de contenidos que se pueden abordar. Por ejemplo, en España existe un importante estudio de comunicación en salud y sería necesario para Chile comenzar a investigar la interacción comunicativa en las diferentes especialidades y conocer las perspectivas de los interlocutores en un contexto hospitalario y, en este caso, la situación comunicativa específica de lo que significa informar y comprender respecto al cáncer. Por ejemplo, Valverde et al. (2014) publicaron el libro: Guía de comunicación oncológica. Estrategias para mantener una buena relación durante la trayectoria de la enfermedad, en el libro se dan recomendaciones para comunicar de forma empática y mantener una relación comunicativa eficaz con el paciente. Desde otra perspectiva, Cuesta et al. (2011) junto a la Fundación Abbot plantean diversos temas, desde el imaginario social de la salud en general, hasta la prevención de drogas. Tales estudios abordan la comunicación en salud desde un interés social y bajo la necesidad de cooperar en el proceso del paciente. Si bien es cierto, esta literatura aporta a nuestros intereses interdisciplinarios no considera las prácticas de salud clínica en nuestra realidad nacional.

\section{Conclusión}

Los principales resultados que se obtienen a partir de la percepción del paciente sobre su interacción comunicativa con el médico tratante, muestra que los pacientes comprenden la información sin importar el nivel educacional que tengan. Existe una cantidad importante de pacientes que opina no haber recibido información respecto de lo que significa: cáncer (46\%) y metástasis (40\%). Cabe recordar que en esta investigación valoró solo la opinión y la voz de los pacientes, por ende, no se puede corroborar que efectivamente no hubo información. Sin embargo, la opinión de los pacientes es clara y se evidencia una comunicación que no fue efectiva. Es por esto, que las técnicas junto con las habilidades de comunicación son necesarias para comenzar a mejorar la entrega de la información durante la interacción comunicativa.

Los pacientes oncológicos están recibiendo de manera constante información por parte del médico tratante, personal de enfermería, los familiares, otros pacientes, internet, entre otras plataformas que afectan el proceso de cáncer. Es importante que la información entregada al paciente sirva para que este interprete su enfermedad y no genere una representación errónea de su realidad a partir de la desinformación o información falsa. De este modo, los pacientes necesitan de una comunicación efectiva durante la interacción comunicativa, para lograrlo los profesionales de la salud deben 
reflexionar sobre sus prácticas y estrategias comunicativas e ir más allá con sus pacientes, involucrarse con ellos y con su familia, ya que cada proceso, paciente y cáncer es diferente, es preciso escuchar y atender a las dudas de ellos.

Es tiempo de preocuparse por el cómo comprende el paciente y según eso acomodar las estrategias comunicativas al contexto socio-situacional. En este sentido, la transparencia del médico tratante y de quienes rodean socialmente al paciente es vital para la comprensión, no solo de los conceptos, sino también de la importancia de su enfermedad y del nuevo estilo de vida que comienza. Asimismo, es importante ampliar a otras especialidades este estudio, como a diabetología, oncología infantil, evaluar las mallas curriculares de los futuros médicos e identificar si les enseñan a cómo comunicar de forma efectiva a los pacientes. La lingüística y la medicina necesitan cooperar mutuamente y pensar en un mismo objetivo: entregar una comunicación efectiva a los pacientes.

\section{Contribuciones y reconocimientos}

No hubo fuentes de financiamiento. Todas las autoras participaron del manuscrito y declaran que no existe ningún tipo de conflicto de interés.

\section{Referencias}

Arraras J, Wintner L, Sztankay M, Tomaszewski K, Hofmeister D, Costantini A, Bredart A, Young T, Kuljanic K, Tomaszewska I, Kontogianni M, Chie W, Kullis D, Greimel E \& Zarandona U (2017). La comunicación entre el paciente oncológico y los profesionales. El Cuestionario de Comunicación de la EORTC. Psicooncología. 14, 107-120.

Camps Herrero C, Gonzales-Cruz V \& Caballero C. (2007). El coste de la mala comunicación. En Comunicación en oncología, pp. 115-133 ed. Sociedad española de oncología médica.

Carrato Mena A. (2007). Prólogo. En Comunicación en oncología, pp. 11-12 ed. Sociedad española de oncología médica.

Caravedo R. (2014). Percepción y variación lingüística. Enfoque sociocognitivo Onomázein 33, 34-38.

Croft W. \& Cruse D. (2008). Lingüística cognitiva. Akal. Madrid.

Herrera A, Ríos M, Manríquez JM \& Rojas G (2014). Entrega de malas noticias en la práctica clínica. Revista médica de Chile. 142, 1306-1315.

Hospital Barros Luco Trudeau (2019). Memorándum N¹22, Resultado de evaluación de Proyecto código 11-12022019. Aprobado por el Comité de Ética Científico S.S.M.S, pp. 1-2.

Hospital Barros Luco Trudeau (2019). Resolución exenta №2326.
Dirección Unidad Proyectos y Estudios Asistenciales S.S.M.S, pp. 1-11. Hospital Barros Luco Trudeau (2018). Planificación estratégica. Accedido en http://www.hospitalbarrosluco.cl/wp-content/uploads/2019/06/ RES_6013_26.12.2018_PE_2018-2021.pdf el 15 de junio de 2020.

Instituto Nacional del Cáncer Chile (2020). Folleto Educativo para pacientes de Radioterapia sobre mama. Accedido en https://www. incancer.cl/wp-content/uploads/2020/04/Educativo-mama.pdf el 17 de diciembre de 2020 .

Instituto Nacional del Cáncer Estados Unidos (2015). ¿Qué es el cáncer?. Accedido en https://www.cancer.gov/espanol/cancer/ naturaleza/que-es el 17 de diciembre de 2020.

Kaplan N. (2004). Nuevos desarrollos en el estudio de la evaluación en el lenguaje: la teoría de la valoración. Boletín de lingüística 22, 52-78.

Kottow M \& Kottow A. (2009). El encuentro clínico: reflexiones semióticas. Revista Bioethicos 3, 194-200

Ministerio de Salud; Subsecretaria de Salud Pública. (2012). LEY NúM. 20.584; Regula los derechos y deberes que tienen las personas en relación con acciones vinculadas a atención en salud. Accedido en https://www.bcn.cl/leychile/navegar?idNorma=1039348 el 17 de diciembre de 2020.

MINSAL (2016). Estrategia nacional de cáncer. Chile 2016. Ministerio de salud, Santiago. Accedido en https://www.minsal.cl/wp-content/ uploads/2016/10/Estrategia-Nacional-de-Cancer-version-consulta-publica.pdf el 17 de diciembre de 2020.

MINSAL (2018). Plan nacional del cáncer 2018-2028. Ministerio de salud, Santiago. Accedido en https://cdn.digital.gob.cl/filer_public/ d3/0a/d30a1f5e-53d9-4a31-a4fe-e90d8d9a2348/documento_plan_ nacional_de_cancer.pdf el 17 de diciembre de 2020.

Moreno Fernández F. (2012). Sociolingüística Cognitiva. Proposiciones, escolios y debates. Iberoamericana Vervuert.

Parodi G. (2008). Géneros del discurso escrito: hacia una concepción integral desde una perspectiva sociocognitiva. En Géneros académicos y géneros profesionales: Accesos discursivos para saber y hacer, pp. 17-37. Ediciones Universitarias de Valparaíso.

Parras D. (2013). La obligación de informar al paciente. Cuestiones sobre el derecho a ser informado. Revista médica de Chile. 141, 1578-1583.

Sentis F. (1997). Interacción y discurso. En Onomázein. 2, 371-386.

Valverde JJ, Gómez M \& Navarrete A. (2014). Guía clínica de comunicación en oncología. Desclée de Brouwer. 